\title{
MAGNETIC FIELD EVOLUTION IN ACCRETING WHITE DWARFS
}

\author{
ANDREW CUMMING \\ Hubble Fellow, Department of Astronomy and Astrophysics, \\ University of California, Santa Cruz, CA 95064, USA
}

\begin{abstract}
I discuss the evolution of the magnetic field of an accreting white dwarf. I show that the ohmic decay time is $7-12$ billion years for the lowest order decay mode, almost independent of core temperature or mass. I then show that the magnetic field structure is substantially altered by accretion if the white dwarf mass increases at a rate $>\dot{M}_{c} \approx(1-5) \times 10^{-10} M_{\odot} \mathrm{yr}^{-1}$. I discuss the implications of this result for observed systems, including the possible evolutionary link between AM Hers and intermediate polars.
\end{abstract}

\section{Introduction}

Calculations of ohmic decay in an isolated, cooling white dwarf show that the magnetic field changes little over its lifetime (Chanmugam \& Gabriel 1972; Fontaine, Thomas, \& van Horn 1973; Wendell, van Horn, \& Sargent 1987). However, an accreting white dwarf may substantially increase its mass on a timescale much shorter than the ohmic decay time, raising the question of what happens to its magnetic field. Here, I describe calculations which show that the field structure may be substantially affected by accretion if the accretion is rapid enough, $\dot{M}>\dot{M}_{c} \approx(1-5) \times 10^{-10} M_{\odot} \mathrm{yr}^{-1}$. At lower accretion rates, ohmic diffusion allows the field to "keep up" with the accretion flow. This critical accretion rate lies in the middle of observed rates, with interesting implications for observations. A detailed account of this work may be found in Cumming (2002).

A major uncertainty in our understanding of accreting white dwarfs is whether the white dwarf mass increases or decreases with time. The answer depends on the amount of mass ejected by classical novae, an un- 
certain quantity both observationally and theoretically. In the calculations presented here, I assume the white dwarf mass increases with time.

\section{Ohmic Decay in Liquid White Dwarfs}

Compressional heating maintains the core of most accreting white dwarfs in a liquid state, with core temperatures $>10^{7} \mathrm{~K}$ (Nomoto 1982). In this case, the ohmic decay timescale is almost independent of white dwarf mass and core temperature.

The timescale for ohmic decay is $t_{\mathrm{ohm}}=4 \pi \sigma L^{2} / c^{2}$, where $\sigma$ is the electrical conductivity, and $L$ the lengthscale over which the magnetic field changes. The larger the conductivity, the less dissipation, giving a longer ohmic time. The insensitivity to core temperature comes about because in a liquid, the electrical conductivity is set by electron-ion scattering, which depends mainly on the electron Fermi energy (and therefore density) rather than temperature, $\sigma \propto \rho^{1 / 3}$ at the center. In contrast, a cooling white dwarf with a solid core has conductivity set by electron-phonon collisions, and the phonon density depends sensitively on temperature. To get the rough scaling with mass, assume a mass-radius relation $R \propto M^{-1 / 3}$, giving a mean density $\rho \propto M^{2}$, and therefore $\sigma \propto M^{2 / 3}$. The scaling with mass then drops out of the ohmic time, $t_{\mathrm{ohm}} \propto \sigma R^{2}=$ constant.

Following the calculations of Wendell et al. (1987) for isolated white dwarfs, I have calculated the ohmic decay modes for detailed models of accreting white dwarfs. This calculation takes into account the variations of $L$ and $\sigma$ at different radii. The lowest order ohmic decay mode has a decay time that varies by a factor of 2 over mass, $\tau=7-12$ billion years.

\section{Calculation of the Critical Accretion Rate}

I adopt two approaches to calculate the critical accretion rate $\dot{M}_{c}$ above which ohmic diffusion can no longer keep up with accretion, and we expect accretion to change the white dwarf field structure. The first, following work on accreting neutron stars (Cumming, Zweibel, \& Bildsten 2001), is to compare local timescales for accretion and ohmic diffusion as a function of depth in the white dwarf envelope. I find that the ratio $t_{\text {diff }} / t_{\text {accr }}$ is insensitive to depth, and is unity for $\dot{M}=(1-5) \times 10^{-10} M_{\odot} \mathrm{yr}^{-1}$.

The second approach is to calculate the global time-dependent evolution of the field under the joint action of accretion and ohmic diffusion. I consider an axisymmetric dipole magnetic field with spherical accretion. The evolution of the field is described by a $1 \mathrm{D}$ advection-diffusion equation which is straightforward to evolve in time. Figure 2 shows the initial and final field configurations after accreting $0.1 M_{\odot}$ onto a $0.6 M_{\odot}$ white dwarf at three different rates. For $\dot{M} \leq 10^{-10} M_{\odot} \mathrm{yr}^{-1}$, the field structure is 
Initial Model

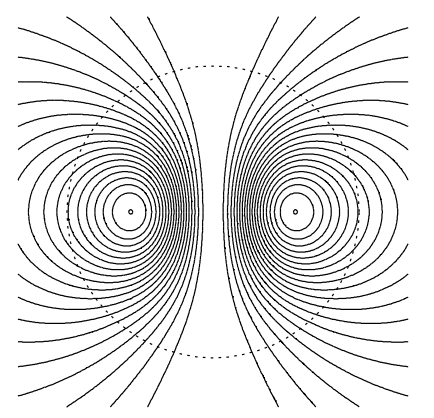

$\dot{\mathrm{M}}=10^{-11} \mathrm{M}_{\odot} / \mathrm{yr}$

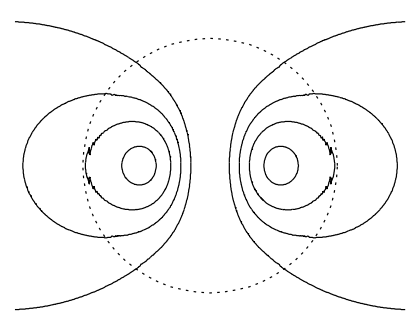

$\dot{\mathrm{M}}=10^{-9} \mathrm{M}_{\odot} / \mathrm{yr}$
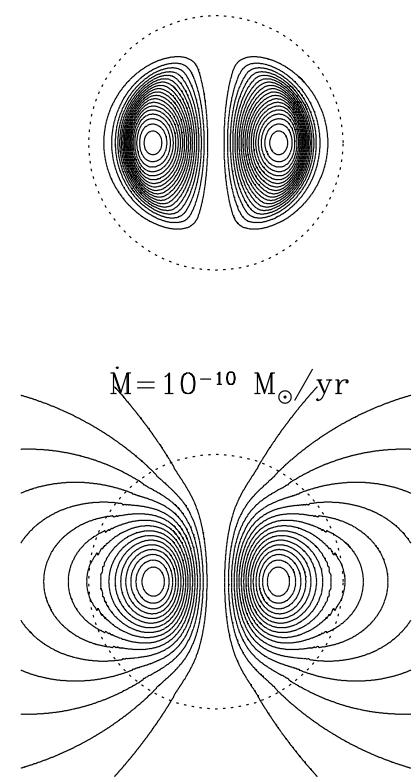

Figure 1. Magnetic field lines before and after accretion of $0.1 M_{\odot}$ onto a $0.6 M_{\odot}$ white dwarf at three different rates, $\dot{M}=10^{-11}, 10^{-10}$, and $10^{-9} M_{\odot} \mathrm{yr}^{-1}$. For $\dot{M} \leq 10^{-10} M_{\odot} \mathrm{yr}^{-1}$, the number density of field lines decreases due to ohmic decay. For $\dot{M}=10^{-9} M_{\odot} \mathrm{yr}^{-1}$, magnetic flux is advected into the interior. Field lines with the same interior flux are shown in each plot.

unaffected by accretion, but the number density of field lines decreases due to ohmic decay. For $\dot{M}=10^{-9} M_{\odot} \mathrm{yr}^{-1}$, however, the field structure is substantially changed as magnetic flux is advected inwards by accretion.

\section{Implications for Observed Systems}

I have shown that the surface magnetic field is reduced by accretion if the white dwarf mass increases at a rate $\dot{M}>(1-5) \times 10^{-10} M_{\odot} \mathrm{yr}^{-1}$. This has a number of implications for observed systems.

An evolutionary connection between AM Hers and intermediate polars? The $\dot{M}$ estimates of Warner (1995) show that AM Hers accrete at low rates, $\dot{M} \approx 5 \times 10^{-11} M_{\odot} \mathrm{yr}^{-1}$, whereas the IPs accrete more rapidly, $\dot{M} \approx(0.2-$ 4) $\times 10^{-9} M_{\odot} \mathrm{yr}^{-1}$. Interestingly, the critical rate $\dot{M}_{c}$ lies between these two values, suggesting the possibility that the magnetic fields in IPs have been reduced by accretion. This would allow for an evolutionary connection 
between IPs and AM Hers, which is suggested by their different orbital period distributions, but has difficulties if $B$ is constant (Hameury et al. 1987; King \& Lasota 1991), since IPs are believed to have weaker fields than AM Hers. A drop in $\dot{M}$, e.g. at $P_{\text {orb }} \approx 3-4$ h would allow the magnetic field to reemerge, on a timescale $\approx 3 \times 10^{8}$ yr $\left(\Delta M / 0.1 M_{\odot}\right)^{7 / 5}(\Delta M$ is the amount of accreted mass), similar to the time for a non-magnetic CV to cross the period gap. The picture is presumably more complex, for example within AM Hers, no correlation is seen between $B$ and orbital period (Wickramasinghe \& Ferrario 2000). However, the fact that the magnetic field may change with time should be kept in mind when considering the evolution of these systems.

Rapidly accreting systems ("Type Ia progenitors") The supersoft X-ray sources and symbiotic systems have $\dot{M} \gg \dot{M}_{c}$, and are believed to be stably burning the accreted matter, increasing the white dwarf mass. We know little about the magnetic fields of these white dwarfs. Pulsations are seen in the symbiotic Z And (Sokoloski \& Bildsten 1999), and a supersoft source in M31 (Osborne et al. 2001; King, Osborne, \& Schenker 2002). If due to magnetic accretion, then $B \approx 10^{7} \mathrm{G}$ in each case. We expect accretion to have a substantial effect on the magnetic field, so these are interesting systems to consider further.

Where are the $10^{9} \mathrm{G}$ accreting white dwarfs? Whereas isolated white dwarf fields extend up to $10^{9} \mathrm{G}$, most AM Hers have $B<10^{8} \mathrm{G}$, with the highest being $2.8 \times 10^{8} \mathrm{G}$ (AM Uma). This may be due to selection effects (Wickramasinghe \& Ferrario 2000). Some difference is to be expected from the different ohmic decay properties - accreting white dwarfs are liquid and have shorter decay times. This could account for a factor of 2 difference over a Hubble time.

I thank D. Wickramasinghe, H. van Horn, and E. Zweibel for useful discussions. This work was supported by NASA Hubble Fellowship grant HF-01138 awarded by the Space Telescope Science Institute, which is operated by the Association of Universities for Research in Astronomy, Inc., for NASA, under contract NAS 5-26555.

\section{References}

Chanmugam G., \& Gabriel M., 1972, A\&A, 16, 149

Cumming, A. 2002, MNRAS, 333, 589.

Cumming, A., Zweibel, E. G., \& Bildsten, L. 2001, ApJ, 557, 958

Fontaine G., Thomas J. H., van Horn H. M., 1973, ApJ, 184, 911

Hameury, J. M., King, A. R., Lasota, J. P., \& Ritter, H. 1987, ApJ, 316, 275

King, A. R., \& Lasota, J. P. 1991, ApJ, 378, 674

King, A. R., Osborne, J. P., \& Schenker, K. 2002, MNRAS, 329, L43

Nomoto, K. 1982, ApJ, 253, 798

Osborne, J. P. et al. 2001, $A \& A A, \mathbf{3 7 8}, 800$

Ritter, H., \& Kolb, U. 1998, $A \mathscr{\mho} A S, \mathbf{1 2 9}, 83$ 
Sokoloski, J. L., \& Bildsten, L. 1999, ApJ, 517, 919

Warner, B. 1995, Cataclysmic Variable Stars, Cambridge Univ. Press, Cambridge

Wendell C. E., van Horn H. M., \& Sargent D. 1987, ApJ, 313, 284

Wickramasinghe D. T., \& Ferrario L. 2000, PASP, 112, 873 